\title{
Narrativa
}

\section{Sud globale e autonomia: narrazioni transculturali, dislocazioni identitarie e sconfinamenti geopolitici nel Cristo si è fermato a Eboli di Carlo Levi}

\section{Vincenzo Binetti}

\section{(2) OpenEdition \\ Journals \\ Edizione digitale \\ URL: https://journals.openedition.org/narrativa/687 \\ DOI: $10.4000 /$ narrativa.687 \\ ISSN: 2804-1224 \\ Editore \\ Presses universitaires de Paris Nanterre}

\section{Edizione cartacea}

Data di pubblicazione: 1 décembre 2017

Paginazione: 103-114

ISBN: 978-2-84016-289-6

ISSN: $1166-3243$

\section{Notizia bibliografica digitale}

Vincenzo Binetti, «Sud globale e autonomia: narrazioni transculturali, dislocazioni identitarie e sconfinamenti geopolitici nel Cristo si è fermato a Eboli di Carlo Levi», Narrativa [Online], 39 | 2017 , online dal 01 décembre 2021, consultato il 14 janvier 2022. URL: http://journals.openedition.org/ narrativa/687 ; DOI: https://doi.org/10.4000/narrativa.687

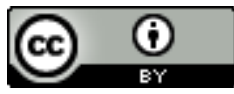

Narrativa est mise à disposition selon les termes de la Licence Creative Commons Attribution 4.0 International. 


\title{
Sud globale e autonomia: narrazioni transculturali, dislocazioni identitarie e sconfinamenti geopolitici nel Cristo si è fermato a Eboli di Carlo Levi
}

\author{
Penso che tutti quelli che vengono dal Sud del mondo rimangono, \\ in un modo o nell' altro, dei clandestini. \\ Mario Fortunato e Salah Methnani, Immigrato \\ - Venite da lontano? \\ - Da Napoli \\ - Emigrante? \\ - No... Sono partito per viaggiare. \\ Massimo Troisi, Ricomincio da tre
}

L

a rappresentazione del Sud nella letteratura italiana solleva importanti questioni identitarie legate a problematiche nozioni politico-culturali di confine, sovranità, cittadinanza, globalizzazione e anche a dislocazioni transculturali di nuove spazialità geo-politiche. Attraverso la rivisitazione di specifiche testualità letterarie diventa possibile, a mio avviso, ripensare e mettere in discussione determinate codificazioni linguistiche e paradigmi concettuali attraverso cui il Sud viene di volta in volta "(re)inventato", mistificato e mitizzato. Tentare di (ri)mappare quindi narrativamente e concettualmente il "meridione" o i meridioni della terra vuol dire allora non solo evitare fuorvianti categorie miranti a visualizzare il Sud come entità uniformante e omogenea, ma provare anche a ripensare nuove linee di confine finzionali e politico-culturali, dando così "spazio" e "autonomia" alla loro continua ibridazione e fluidità e quindi al loro valore potenzialmente conflittuale e insorgente.

Oggi vorrei soffermarmi brevemente su un testo che di per sé "sfugge a qualsiasi rigida classificazione [perché] è insieme reportage, diario, cronaca, 
romanzo sociale, narrazione lirica, ecc."1, e cioè Cristo si è fermato ad Eboli, di Carlo Levi', scritto tra il dicembre del '43 e il luglio del '44 e pubblicato da Einaudi nel 1945, non per addentrarmi in coeve diatribe "novecentesche" legate alla ricezione e al contributo del Cristo rispetto alla questione meridionale, basti pensare alle critiche mosse da chi, come Alicata, vedeva a suo tempo nel romanzo "l'enunciazione di una serie di tesi senza consistenza teorica" e un'estraneità misticheggiante a "ogni proposito di spiegare storicisticamente le ragioni dell'inferiorità sociale del Mezzogionno"3, ma per tentare invece di capire come esso possa servici oggi a problematizzare il Sud Italia (o i vari sud del pianeta) all'interno di un discorso globale.

Cosa significa allora ripensare nella contemporaneità all'ormai irrimediabilmente mutata e forse inesistente Lucania del romanzo leviano? Esistono, in altre parole, in questo conflittuale e a volte anche contraddittorio racconto di viaggio tra il Nord e il Sud d'Italia - percorso geografico-esistenziale, tra l'altro, quello di cui si fa portavoce il protagonista, così fortemente intimista e privato, ma allo stesso tempo segnato da ineludibili e significativi momenti di aggregazione comunitaria - delle potenziali implicazioni stilistico-letterarie e criticoteoriche legate a riflessioni e pratiche politiche odierne potenzialmente destabilizzanti ed innovative rispetto alla nostra riflessione sul sud globale?

Il perno concettuale intorno al quale vorrei, sia pur brevemente, provare a rileggere il testo leviano è quello dell'autonomia o forse meglio di una "postautonomia": non solo infatti l'autonomia legata alle riflessioni politiche che lo stesso Levi elaborava in chiave gobettiana all'interno delle pagine di Giustizia e Libertà, ma anche, e perché no, quella che, ad esempio, lo stesso Franco Berardi sostiene possa servirci oggi come "autoregolazione del corpo sociale, nella sua indipendenza e nelle sue interazioni con la norma disciplinare": "il problema che ci dobbiamo porre - scrive infatti Bifo - è quello dell'autonomia dello spazio sociale dal dominio capitalistico $[\ldots]$ autonomia significa $[\ldots]$ lotta, ritirata, alienazione, sabotaggio, linee di fuga dal sistema di dominio" ". Ed è proprio alla

1. La Porta, Filippo, "Carlo Levi e la nuova narrativa meridionale", in De Donato, Gigliola (a cura di), Verso $i$ sud del mondo. Carlo Levi a cento anni dalla nascita. Roma, Donzelli, 2003, p. 171.

2. Levi, Carlo, Cristo si è fermato a Eboli, Torino, Einaudi, 1945.

3. Alicata, Mario, "Il meridionalismo non si può fermare a Eboli", in Cronache meridionali, n. 5, 1954, ripreso nella ristampa Einaudi 1991 del Cristo si è fermato a Eboli (la cit. è a p. 240).

4. Berardi, Franco, "Che significa oggi autonomia?", in http://www.republicart. net/disc/realpublicspaces/berardi01_it.pdf, settembre 2003, p. 2.

5. Ibid., p. 1. 
luce di questa possibile rilettura in chiave "autonoma" e forse anche "impolitica" del romanzo ${ }^{7}$ che vorrei offrire in questa sede una disanima sulla questione del sud lucano, e non solo, come riferimento a un concetto di autonomia rispetto alla logica neo-liberista di un nord planetario sempre più egemonico e globalizzante.

Ora, come dicevo, Levi aveva già anticipato nel 1932 nei quaderni di Giustizia e Libertà, prima ancora del Cristo, quelle che a suo avviso dovevano essere le proposte programmatiche "autonomiste" per una politica culturale e una pratica rivoluzionaria volte a scardinare alle fondamenta la centralità autoritaria $\mathrm{e}$ omologante dello Stato nazione italiano:

Parlare di autonomia nel puro senso è affermare il valore morale della politica; intendere che la lotta politica deve essere in Italia lo strumento di un rinnovamento di civiltà $[\ldots]$. Tutto il programma va letto e interpretato secondo questo principio, sia esso chiamato col suo nome, autonomia, o con i termini equivalenti di Rivoluzione, Democrazia, ecc., o con quello di azione spontanea delle masse operaie e contadine [...]. Il problema in Italia è particolarmente arduo: problema di rivoluzione in un paese senza eredità politiche $[\ldots]$ dobbiamo creare uno Stato con i mezzi dell'anarchia .

Ma al di là del valore prettamente programmatico di queste proposte politiche che affrontavano in maniera consapevole la necessità e l'urgenza di rifiutare la repressione autoritaria dello Stato fascista e erano chiaramente frutto del clima politico-culturale in cui furono pensate, mi sembra sintomatico che esse anticipino in qualche modo il tentativo leviano di trasformare il suo confino al sud in un'occasione per valorizzare le potenzialità "anarchiche" di "esodo" e autonomia di queste comunità contadine non solo rispetto allo Stato, ma anche rispetto alla Storia e quindi ad un ripensamento spazio-temporale ed ontologico dell'essere "non-umano":

6. Cfr, anche, a questo proposito: LA PorTA, Filippo, "Carlo Levi. Liberarsi della politica attraverso la politica”, in De Donato, Gigliola (a cura di), Oltre la paura. Percorsi nella scrittura di Carlo Levi, Roma, Donzelli, 2008.

7. Come ci ricorda Massimo Cacciari nella sua lettura in chiave nietzschiana di questo concetto: "Norm and law point to the universal method on whose basis every subject becomes totality. The unpolitical is the work of deconstruction of this totality...the unpolitical represents the critique of values on whose bases alone such totality is conceivable...the task of the democratic idea consists in the desacralization of the political" (CAcciari, Massimo, The Unpolitical. On the Radical Critique of Political Reason, New York, Fordham University Press, 2009, pp. 96-98).

8. Levi, Carlo, Il dovere dei tempi. Prose politiche e civili, Roma, Donzelli, 2004, pp. 24-27. 
Sono passati molti anni, pieni di guerra, e di quello che si usa chiamare Storia. $\mathrm{Ma}[\ldots]$ mi è grato riandare con la memoria a quell'altro mondo $[. .$.$] negato alla$ Storia e allo Stato... a quella mia terra senza conforto e dolcezza, dove il contadino vive [...] la sua immobile civiltà. Noi non siamo cristiani - essi dicono, - Cristo si è fermato a Eboli. Cristiano vuol dire, nel loro linguaggio, uomo [...]. Noi non siamo cristiani, non siamo uomini, non siamo considerati come uomini, ma bestie [...]. Cristo non è mai arrivato qui, non vi è arrivato il tempo $[\ldots]$ né il legame tra le cause e gli effetti, la ragione e la Storia?

Ed è proprio questo utilizzo di un linguaggio "minore" che permette a Levi di raccontare la "storia" di una comunità certamente consapevole della propria marginalità, ma desiderosa di intraprendere una sorta di "conversazione" transculturale capace di infrangere il muro della separazione identitaria tra nord e sud voluto dalle logiche sovrane dello stato nazione e stabilire invece un processo costituente di ibridazione e di contaminazione reciproca. Sintomatico a questo proposito è l'incontro tra il protagonista e il becchino del villaggio durante il quale, appunto, l'utilizzo di un "gergo oscuro" e "arcano", di un codice comunicativo altro, permette tuttavia lo scambio e la familiarizzazione tra due mondi all'apparenza irrimediabilmente diversi e separati tra loro e il superamento dell'intraducibilità ${ }^{10}$ linguistica e culturale iniziale tra il nord dell'intellettuale torinese e il sud dei contadini lucani:

Questo essere indefinibile... era insieme il becchino e il banditore comunale...Quel vecchio aveva un potere arcano, era in rapporti con le forze sotterranee, conosceva gli spiriti, domava gli animali...era l'incantatore dei lupi [...]. Per il vecchio le ossa, i morti, gli animali e i diavoli erano cose familiari, legate, come lo sono del resto, qui, per tutti, alla semplice vita di ogni giorno. - Il paese è fatto delle ossa dei morti, - mi diceva, nel suo gergo oscuro, gorgogliante come un'acqua sotterranea che esca improvvisamente fra le pietre; e faceva... una smorfia che forse era un sorriso ${ }^{11}$.

Se dunque la "consapevolezza della molteplicità dei linguaggi è la condizione necessaria per iniziare a comprendere la complessità del mondo che

9. Levi, Carlo, Cristo si è fermato a Eboli, cit., p. 3.

10. Scrive Chambers a proposito: "Forse, allora, la traducibilità diviene una parola molto più preziosa nei suoi rimandi, poiché non riguarda solo la delicata relazione tra sistemi culturali e linguistici diversi, e sistemi del sapere diversi, ma l'intima riflessione sulla modernità come complesso e intricatissimo gioco di inclusioni ed esclusioni dallo spazio e dal tempo della ragione occidentale" (CHAmbers, Iain [a cura di], Esercizi di potere. Gramsci, Said e il postcoloniale, Roma, Meltemi, 2006, p. 44).

11. Levi, Carlo, Cristo si è fermato a Eboli, cit., pp. 60-61. 
ci circonda"12, allora è forse possibile vedere nel tentativo autoriale di dare visibilità a uno spazio comunitario autonomo, il bisogno di valorizzare le potenzialità politiche destabilizzanti e costituenti della sua intrinseca alterità, riconoscendo, allo stesso tempo, come ci ricorda Cassano, che oggi "iil mondo contadino di Levi continua in tutti quegli uomini e quelle donne che, arrivando da altre terre, relativizzano le nostre certezze"13. Ed è appunto questa potenzialità antagonista di una comunità "straniera" situata ai confini di uno Stato fascista che tuttavia "impone goffamente la sua presenza esteriore" 14 - basti pensare alla visualizzazione simbolica del "monumentale pisciatoio"15 eretto al centro della piazza di Gagliano - a spingere i suoi abitanti, soggettività autonome senza cittadinanza che problematizzano la nozione sovrana dell'essere cittadino ${ }^{16}$, a rinnegare Roma e le sue guerre nazionali come spazio urbano emblematicamente rappresentativo di uno Stato "straniero" e "malefico":

Per la gente di Lucania, Roma non è nulla: è la capitale dei signori, il centro di uno Stato straniero e malefico [...] non soltanto gli scopi, ma anche la condotta della guerra riguardava quell'altra Italia, di là dai monti, e aveva poco a che fare con i contadini [...]. Anche quella era stata una guerra di Roma. Anche allora si seguivano i tre colori, che qui sembrano strani, i colori araldici di un'altra Italia, incomprensibile, volontaria e violenta ${ }^{17}$.

Ecco perché anche il brigantaggio diventa allora, nel testo leviano, guerra contadina, esplosione momentanea e rivoltosa di "eroica follia", seppur disperata e senza speranza di vittoria o di velleitarie prese di potere, ma comunque

12. Cassano, Franco, "Cinquantasei anni dopo "Cristo", in De Donato, Gigliola, D’Amaro, Sergio (a cura di), Carlo Levi e il Mezzogiorno, Foggia, Claudio Grenzi Editore, 2003, p. 15.

13. Ibid., p. 16.

14. Mastrangelo, Luigi, Società e politica nelle "Italie" di Carlo Levi, Napoli, Luciano Editore, 2014, p. 17.

15. "In mezzo alla piazza si ergeva uno strano monumento, alto quasi quanto le case, e, nell'angustia del luogo, solenne ed enorme. Era un pisciatoio: il più moderno, sontuoso, monumentale pisciatoio che si potesse immaginare" (Levi, Carlo, Cristo si è fermato a Eboli, cit., p. 40).

16. Vedi, a proposito, Jean Casimir e la sua nozione di "inhabitants" che "calls into questions the imperial notion of 'citizen'. Inhabitants are those who name the dwelling place, those who in-habit a place, a land and not those who depend on the law and the rights dictated by a State that leaves the inhabitants out of its citizens" (citato in Levander, Caroline, Mignolo, Walter, "Introduction: The Global South and World Dis/Order", in The Global South, vol. 5, n. 1, 2011, p. 5).

17. Levi, Carlo, Cristo si è fermato a Eboli, cit., pp. 108 e 117-119. 
espressione significativa di un "discorso quotidiano" e familiare di disubbidienza, attraverso il quale dar voce al proprio desiderio comunitario di insubordinazione nei confronti dello Stato e di rifiuto delle sue leggi:

I contadini erano tutti dalla parte dei briganti, e, col passare del tempo, quelle gesta che avevano così vivamente colpito le loro fantasie, si sono indissolubilmente legate agli aspetti familiari del paese, sono entrate nel discorso quotidiano, con la stessa naturalezza degli animali e degli spiriti, sono cresciute nella leggenda e hanno assunto la verità certa del mito...I briganti difendevano, senza ragione e senza speranza, la libertà e la vita dei contadini, contro lo Stato, contro tutti gli Stati [...]. Col brigantaggio la civiltà contadina difendeva la propria natura, contro quell'altra civiltà che le sta contro e che, senza comprenderla, eternamente la assoggetta: perciò, istintivamente, i contadini vedono nei briganti i loro eroi ${ }^{18}$.

Queste pratiche quotidiane imprevedibili e destabilizzanti, siano esse individuali o collettive, reali o mitologiche, magiche o razionali, che contraddistinguono l'“inoperosa comunità" - per dirla con Nancy - di queste soggettività operanti comunque ai margini, sul borderline di una spazialità costantemente in bilico tra il dentro e il fuori della sovranità nazionale, tra un "noi" familiare e un "loro" estraneo e incomprensibile, insomma tra un'umanità cristiana del nord e un sud "disumano" e senza Cristo perché emblematicamente relegato al di là di Eboli, finiscono per complicare problematicamente anche la percezione del Tempo della Storia, di ciò che dovrebbe contraddistinguere la consequenzialità lineare e prevedibile dell'evolversi cronologico di una temporalità oggettivamente e pubblicamente condividibile. Ecco perché l'orologio del protagonista, che attende solo nella sua cucina la fine dell'anno, subisce sul finire del romanzo un simbolico arresto:

Arrivammo alla fine dell'anno. Volli attendere la mezzanotte secondo l'usanza...Il mio orologio si era fermato, e nessun ritocco di fuori poteva giungermi e indicarmi il passare del tempo, dove il tempo non scorre. Così finì, in un momento indeterminato, l'anno 1935, quest'anno fastidioso, pieno di noia legittima, e cominciò il 1936, identico al precedente, e a tutti quelli che sono venuti prima, e che verranno poi, nel loro indifferente corso disumano ${ }^{19}$.

18. Ibid., pp. 121-123.

19. Ibid., p. 182. 
Ma è proprio nella dimensione "cairologica", direi anche bergsoniana, di questa imprecisabile indeterminatezza temporale, che diventa forse possibile stabilire, al di là di una indifferente disumanità, una diversa socialità comunitaria: "the experience of timelessness is the result of a return to an undifferentiated sense of collectivity" ${ }^{20}$ che però non significa una facile e improponibile rinuncia escapista in un mondo "naturale", astratto e misticheggiante fuori dal Tempo della Storia, ma appropriazione autonoma e, allo stesso tempo, linea di fuga e autogoverno consapevole "di un mondo singolare di socialità" ${ }^{21}$ all'interno di questa stessa comunità e attraverso la sua alterità spazio-temporale.

$\mathrm{Ed}$ è in questa prospettiva che a mio avviso andrebbe (re)interpretato anche il valore semantico-temporale del "crai" contadino di cui ci parla Levi in alcune pagine del suo romanzo; non per confermare, ancora una volta, un'eventuale e prevedibile atemporalità mitologica peculiare a questa comunità incapace di adeguarsi allo scandirsi quotidiano del Tempo della Storia, ma per valorizzare invece, pur attraverso ineludibili contraddizioni e problematicità, l'aspetto trasgressivo della propria appartenenza a una temporalità "altra" e il valore conflittuale e destrutturante della propria soggettivizzante quotidianità:

Il mutarsi dei giorni era un semplice variare di nuvole e di sole...Tutto il domani, fino alla fine dei tempi, tendeva a diventare anche per me quel vago "crai" contadino, fatto di vuota pazienza, via dalla storia e dal tempo. Come talvolta il linguaggio inganna, con le sue interne contraddizioni! In questa landa atemporale, il dialetto possiede delle misure del tempo più ricche che quelle di alcuna lingua; di là da quell'immobile, eterno crai, ogni giorno del futuro ha un suo proprio nome. Crai è domani, e sempre...Queste parole [...] sono come una riprova della inutilità di voler distinguere nelle eterne nebbie del crai ${ }^{22}$.

Una misura del tempo dunque, come sottolinea lo stesso Levi, decisamente "più ricca" che si esprime e si autodefinisce attraverso una gergalità "locale" e de-centrata che sembra però quasi sollevare il protagonista dalla "pesantezza" del confino e sottrarlo seppur momentaneamente alle rigide categorie comportamentali imposte dallo Stato fascista: una linea di fuga, insomma, che si espleta, ancora una volta, anche attraverso una sorta di calviniana "leggerezza" spaziotemporale che gli permette simbolicamente di "volare, senza peso, come un

20. Farrell, Joseph (a cura di), The Voices of Levi, Bern, Peter Lang, 2007, p. 105.

21. Berardi, Franco, "Cosa significa oggi autonomia?”, cit., p. 6.

22. Levi, Carlo, Cristo si è fermato a Eboli, cit., pp. 184-185. 
uccello" 23 e di sentirsi come "staccato da ogni cosa, da ogni luogo, remotissimo da ogni determinazione, perduto fuori dal tempo, in un infinito altrove"24. Ed è proprio la consapevolezza di poter in qualche modo entrare "nel cuore stesso del mondo" 25 e divenire, allo stesso tempo, parte attiva di un'esperienza comunitaria non istituzionalizzata, proprio perché transitoria e costituente, a capovolgere e destabilizzare ontologicamente la rigida costruzione identitaria del suo essere confinato torinese nelle terre straniere di un sud all'apparenza inaccessibile perché relegato dallo stato nazione nel non-luogo dell'eccezionalità e a dare spazio invece ad una soggettività insorgente e partecipe pervasa da una imprevedibile e antagonista "felicità immensa [e dal] senso fluente di una infinità pienezza"26.

Il romanzo si conclude con quelle che, come sappiamo, rimangono le pagine forse più politicamente consapevoli del testo leviano, dove l'autore sembra voler emblematicamente rimandare il lettore ai toni programmatici di "Giustizia e Libertà" ${ }^{27}$; ma quello, che a mio avviso, rimane fondamentalmente significativo in queste parole di commiato autoriale è l'implicito richiamo a una potenziale rivisitazione della questione meridionale, del rapporto tra Nord e Sud d'Italia, ma anche e soprattutto dei meridioni del pianeta e la messa in discussione radicale della percezione aleatoria e normativa che di essi ci offre la strategia egemonica della "religione statale" e la logica uniformante del potere globale. Scrive, infatti, Levi:

Senza una rivoluzione contadina, non avremo mai una rivoluzione italiana, e viceversa. Le due cose si identificano. Il problema meridionale non si risolve dentro lo Stato attuale, né dentro quelli che, senza contraddirlo radicalmente, lo seguiranno. Si risolverà soltanto fuori di essi, se sapremo creare una nuova idea politica e una nuova forma di Stato...Bisogna che noi ci rendiamo capaci di pensare e di creare un nuovo Stato, che non può più essere né quello fascista, né quello liberale, né quello comunista, forme tutte diverse e sostanzialmente identiche della stessa religione statale ${ }^{28}$.

23. Ibid., p.197.

24. Ibid., p. 198.

25. Ibid., p. 199.

26. Ibid.

27. Cfr. Ibid., pp. 219-223.

28. Ibid., pp. 222-223. 
Ecco perché, in questa prospettiva, Levi riesce a spostare la sua riflessione politica, maturata in "un anno di vita sotterranea" 29 , nella direzione certamente innovativa e provocatoria di una messa in discussione radicale e di un "ripensamento" ontologico, non solo del concetto di Stato nazione e di mistificanti costruzioni geopolitiche e culturali del Sud, ma anche e soprattutto di ormai improponibili categorie politiche e giuridiche che definiscono il concetto di individuo in quanto entità chiusa in se stessa e per questo non-relazionale; un "capovolgimento" della politica che in quanto tale possa avviare una ridefinizione dei rapporti e delle relazioni tra individuo, Stato e comunità; una forma "costituente" che, come sostiene in maniera inequivocabile lo stesso Levi, possiamo chiamare appunto autonomia:

Dobbiamo ripensare ai fondamenti stessi dell'idea di Stato: al concetto di individuo che ne è la base; e, al tradizionale concetto giuridico e astratto di individuo, dobbiamo sostituire un nuovo concetto, che esprima la realtà vivente, che abolisca la invalicabile trascendenza di individuo e di Stato. L'individuo non è un'entità chiusa, ma un rapporto, il luogo di tutti i rapporti...Questo capovolgimento della politica... questa strada si chiama autonomia ${ }^{30}$.

Questa "realtà vivente", questa autonomia di tutte le "forme della vita sociale" 31 di cui ci parla Levi implica perciò anche una visione più ampia e problematica del Sud che nella contemporaneità significa soprattutto riconoscere proprio in quel confine immaginario che passa da Eboli e "separa il Profondo Sud dal resto del paese...una linea mobile, semovente, [che] oggi attraversa le nostre città...e si ritrova a ogni latitudine del globo e perfino dentro i singoli individui" 32 . Un sud insomma che, come suggerisce La Porta, è "migrante, come i suoi molti (e spesso disastrati) abitanti" ${ }^{33}$ e quindi non può e non deve essere relegato, secondo le categorie e i modelli di una cartografia pianificata dall'ordine globale, nella immobilità omologante di uno spazio confinato e facilmente catalogabile e assimilabile; un sud che riconosce invece proprio nella

29. Ibid., p. 223.

30. Ibid.

31. Scrive ancora Levi a tal proposito: "Lo Stato non può essere che l'insieme di infinite autonomie, una organica federazione. Per i contadini, la cellula dello Stato, quella sola per cui essi potranno partecipare alla molteplice vita collettiva, non può essere che il comune rurale autonomo [...]. Ma l'autonomia del comune rurale non potrà esistere senza l'autonomia delle fabbriche, delle scuole, delle città, di tutte le forme della vita sociale" (Ibid.).

32. La Porta, Filippo, "Carlo Levi e la nuova narrativa meridionale", cit., p. 172.

33. Ibid., p. 168. 
sua mobilità, nella "consapevolezza della propria autonomia etica e civile" ${ }^{34}$, il potenziale innovativo e conflittuale di resistenza (o "re-existance" secondo l'accezione di Adolfo Albán Achinte ${ }^{35}$ ) rispetto a un discorso universalizzante volto a colonizzare e inglobare nel proprio nomos ogni diversità, sminuendone così qualsiasi implicito slancio antagonista e insubordinante.

Il racconto leviano sulla Lucania si inserisce inoltre provocatoriamente all'interno di un'importante riflessione politica e teorica sul "pensiero meridiano" per dirla con Cassano - e sul valore deterritorializzante che queste soggettività comunitarie, nomadiche e diasporiche, assumono nel processo continuo di rimappatura geopolitica e transnazionale del mediterraneo, permettendo, allo stesso tempo, una messa in discussione necessaria di un concetto impermeabile e categorico di stato-nazione e del linguaggio letterario e culturale che lo celebra e lo sancisce: "L'autonomia del sud deve essere [invece] in primo luogo autonomia teorica e politica...il riconoscimento della dimensione mediterranea del Mezzogiorno [...] luogo di un nuovo incontro tra i popoli e le culture" 36 , anche perché, ci ricorda ancora giustamente Cassano, il Sud non può essere "il 'non ancora' della modernità, ma ciò che consente di pensare al di là di essa, di costruire un multiverso difficile e necessario" ${ }^{\prime 37}$.

Levi dunque propone ai suoi lettori, "attraverso una scrittura nomade, a-sistemica e impura" 38 e forse anche come conseguenza di una sua soggettiva "condizione a-polide e cosmopolita di un sapere e di una pratica politica che vivevano nello status permanente di esilio in patria" 39 , una testualità letteraria che diventa però, allo stesso tempo, discorso consapevole della propria alterità, e per questo trasgressivo e "militante", potenzialmente capace di trasformare l'apparente "arretratezza" di quella realtà contadina relegata ai confini meridionali della modernità, in una espressione, seppur transitoria, di autogoverno e di contropotere: "non tanto un mondo da redimere, da restituire al progresso,

34. Piperno, Franco (a cura di), Vento del meriggio. Insorgenze urbane e postmodernità nel Mezzogiorno, Roma, DeriveApprodi, 2008, p. 7.

35. Citato in Levander, Caroline, Mignolo, Walter, "Introduction: The Global South and World Dis/Order", cit., p. 10.

36. Cassano, Franco, "Cinquantasei anni dopo 'Cristo", cit., pp. 12-13.

37. Ibid., p. 213.

38. Moliterni, Fabio, "L'antico nel nuovo. Carlo Levi e il 'pensiero vivente", in Giannone, Antonio Lucio, Cristo si è fermato a Eboli di Carlo Levi, Pisa, Edizioni ETS, 2015, p. 85.

39. Ibid., p. 80. 
quanto un modello alternativo di civiltà, e cioè un'alternativa all'homo oeconomicus della civiltà borghese, urbano-industriale, al culto della storia"40.

Forse, in fin dei conti, si potrebbe immaginare provocatoriamente, rileggendo oggi il testo leviano, la storia di un Cristo che "fortunatamente" si è fermato a Eboli: ribaltare insomma la velleitaria speranza consolatoria e accomodante di una "non-umanità" meridionale sperabilmente redenta da una salvifica e ipotetica cristianizzazione in chiave umanistica di un Nord rigorosamente e omogeneamente "civile" e moderno e pensare invece all'implicito valore dirompente e indisciplinato di un Sud che "spiazzando ogni nostra aspettativa e vanificando ogni bussola di orientamento" "41, asserisce l'intransigente autonomia della sua spazialità geopolitica, linguistica e culturale. Se infatti vogliamo pensare oggi al sud globale come a un "emergent dis/ordering system...a place of struggle between, on the one hand, the rethoric of modernity and modernization... and, on the other, the struggle for independent thought and decolonial freedom" ${ }^{42}$, allora dobbiamo ripensare in maniera radicale a una diversa cartografia planetaria che vede proprio nella mobilità necessaria dei suoi confini, nella ibridazione di questa ormai improponibile e rigida demarcazione spaziotemporale tra Nord e Sud, una potenzialità politica liberatoria ${ }^{43}$ e una pratica militante di autodeterminazione e di insubordinazione. Se inoltre globalizzazione oggi vuol dire, anche e soprattutto, come sostiene Carlo Galli, "mobilitazione globale" 44 e cioè "sconfinamento, sfondamento di confini, deformazione di geometrie politiche" ${ }^{45}$, allora queste spazialità transitorie e costituenti ${ }^{46}$ di autonomia comunitaria, abitate da soggettività nomadiche e "migranti", antagoniste rispetto al progetto egemonico e alle categorie geopolitiche dello Stato nazione

40. La Porta, Filippo, “Carlo Levi e la nuova narrativa meridionale”, cit., p. 115.

41. La PorTa, Filippo, Narratori di un sud disperso, Napoli, L'ancora del mediterraneo, 2000, p. 114.

42. Levander, Caroline, Mignolo, Walter, "Introduction: The Global South and World Dis/Order", cit., pp. 2-4.

43. Scrive infatti Carlo Galli a proposito: “oggi probabilmente la libertà sta nel cercare nuovi confini: non certo per realizzare chiusure dettate dalla paura dell'aperto, o dei nuovi incontri, ma come nuove linee-guida orientative, che delimitano gli spazi in cui i nuovi incontri delle nuove soggettività con se stesse e con gli altri divengano possibili e sensati' (Galli, Carlo, Spazi politici. L'età moderna e l'età globale, Bologna, Il Mulino, 2001, p. 15).

44. Ibid., p. 147.

45. Ibid., p. 133.

46. Sostiene ancora GALLI: "L'impegno [...] a immaginare un processo costituente in chiave federale, capace di organizzare una costituzione le cui istituzioni 'costituite' non pretendano di esaurire e rinchiudere le energie 'costituenti”' (Ibid., pp. 171-172). 
e del potere globale, rappresentano forse possibili strategie di resistenza e di lotta: "Queste soggettività hanno il proprio modello nei migranti, ma, a differenza di molti di questi, non chiederebbero inclusione e integrazione, quanto piuttosto mobilità, possibilità di non adesione, di secessione, di lotta (termine più appropriato che non 'rivoluzione'), di cittadinanze plurime, a tempo, parziali" ${ }^{47}$.

Ecco infine perché il viaggio di ritorno verso il nord Italia con cui si conclude il romanzo leviano rappresenta non solo l'ormai implicita "estraneità" dell'autore rispetto a precedenti categorie identitarie di appartenenza, cioè la constatazione consapevole del valore trasgressivo del suo essere soggettività in bilico perché irrimediabilmente contaminata e destabilizzata dall'esperienza al sud, ma anche testimonianza emblematicamente antesignana di un discorso politicoculturale relativo alle rappresentazioni dei sud, dei suoi confini e delle sue continue dislocazioni identitarie che si ripropone oggi problematicamente e in maniera conflittuale nelle scritture "migranti" di autori contemporanei. Un viaggio, in altre parole, che, come quello di Levi in Lucania, ma anche, per fare un esempio più recente, come quello di Salah Methnani ${ }^{48}$ dalla Tunisia all'Italia, non si traduce nella pre-codificata verticalità di un viaggio di emancipazione e integrazione dal sud "africano" al nord "europeo", né viceversa nell'altrettanto riappacificante viaggio di ritorno dal nord industrializzato e alienante al sud pre-moderno e per questo consolatorio, ma va anche assunto, proprio a causa della sua "circolarità" e trasversalità e della sua autonoma mobilità e ibridazione, come "passaggio" e cioè, come sostiene giustamente Giacomo Marramao, "nel duplice significato di viaggio e di mutamento, di rischio e di opportunità [per] negoziare un nuovo spazio comune, costruire insieme una nuova casa dell'universale...e scorgere neglialtriun'autonoma eoriginale prospettivauniversalizzante" ${ }^{\prime 49}$.

Vincenzo BINETTI University of Michigan

47. Ibid., pp. 160-161.

48. Con queste parole si conclude infatti emblematicamente il romanzo: "L'autobus imboccò la grande strada per El Fahs e Tunisi. Cercai nella valigia, allungata sotto il sedile davanti a me, il quadernetto giallo. Scrissi poche parole... Pensai che il viaggio cominciava adesso" (Fortunato, Mario, Methnani, Salah, Immigrato, Milano, Bompiani, 2006, pp. 129-130).

49. Marramao, Giacomo, Passaggio a Occidente. Filosofia e globalizzazione, Torino, Bollati Boringhieri, 2009, pp. 10, 255 e 265. 